\title{
Scope of herbal disinfectants to fight against SARS-CoV2 virus
}

\author{
Madhurya, L. ${ }^{1}$, Urvashisahu ${ }^{1,2}$, Ezhilvendan, S. ${ }^{1,2 *}$, Sumithradevi, S. ${ }^{1,2}$, Vivekbabu, C.S. ${ }^{1,2}$ \\ ${ }^{1}$ Food Protectants and Infestation Control Department, CSIR-Central Food Technological Research Institute, Mysore - 570020 , India \\ ${ }^{2}$ Academy of Scientific and Innovative Research (AcSIR), Ghaziabad - 201 002, India \\ *Corresponding author: ezilvendan@cftri.res.in
}

\section{ARTICLE HISTORY}

Received: 20 July 2021

Revised: 16 December 2021

Accepted: 16 December 2021

Published: 31 December 2021

\begin{abstract}
Globally, COVID-19 outbreak is a major social issue in the current trend. SARS-CoV2 is a novel coronavirus causing Severe Acute Respiratory Syndrome in human and it is spreading rapidly among human population. In order to prevent SARS-CoV2 infection and managing this corona disease, WHO formula based alcoholic hand sanitisers are being widely used as one of the primary preventive agent and the demand is increasing worldwide. Herbal extracts and/or their phytochemicals have been considered as natural sources for formulating herbal hand sanitizers as alternative to alcoholic products. In this correspondence, we have described about the probable mechanistic action of herbal bioactives to fight against COVID-19 virus. Understanding of mechanistic action of bioactives could be useful to formulate herbal hand sanitizers and the products have high demand in the global sanitizer market.
\end{abstract}

Keywords: COVID-19; SARS-CoV2; herbal sanitizer; herbal disinfectant; phytochemical bioactive.

\section{INTRODUCTION}

Currently, coronavirus disease (COVID-19) outbreak is a global health issue, which originated from the Wuhan city, China. This disease caused by the novel "Severe Acute Respiratory Syndrome Coronavirus 2 (SARS-CoV-2)" and it is spreading among the human population. The SARS-CoV2 primarily affects respiratory system and the symptoms vary from mild dry cough to severe chest pain, and the virus is highly contagious. Since December 2019, the COVID-19 incidence rate is drastically increasing all over the world. In India, first case of COVID-19 was recorded in Kerala state on February 2020 and has widely spread into most of the states in the country day by day. The World Health Organization (WHO) has declared the COVID-19 outbreak as a pandemic disease. Globally, the increased risk of emergence of variants, WHO promoted the characterization of specific Variants of Concern (VOCs), Variants of Interest (VOIs) and Variants Under Monitoring (VUMs) in order to monitoring and management of COVID-19 pandemic. Accordingly as of November 2021, a number of variants have been identified and characterized as VOCs (Alpha, Beta, Gamma, Delta, and Omicron), VOIs (Lambda and Mu) and VBMs (> 10 vairants) (WHO, 2021). Previously, SARS-CoV-1 outbreak was recorded in 2003 with $\sim 8000$ cases and $\sim 800$ deaths worldwide and was successfully managed (Wilder-Smith et al., 2020). Whereas, the prevention and management of COVID-19 incidence is a global challenging task in the current trend.

The WHO and Indian Council of Medical Research (ICMR) governing bodies provided several guidelines of preventive measures for the management of COVID-19. Since asymptomatic cases are also being reported, as a precautionary and control measure, self-awareness and personal hygiene becomes imperative. Accordingly, hand sanitizer applications are playing a vital role as one of the major preventive measures against the spreading of coronavirus infections. With the increase in demand, some of the scientific organizations (e.g., CSIR, IITs, IISc, DBT, ICMR, Universities, etc.,) in India had actively participated in bulk production of alcohol based hand sanitizers (ABHS) by following the formula of WHO (2010) and supplied to the local public. On the other hand, the demand for non-alcohol based hand sanitizer formulations (NAHS) is on increase to minimize demand of ABHS. Typically, the ABHS formulations may contain $60-90 \%$ alcohol (ethanol) and the NAHS formulations may contain benzalkonium chloride or triclosan as a major disinfectant component. Frequent use of both ABHS and NAHS formulations may cause mild level of adverse effects rarely in human beings. Alcohol based disinfectants (ethanol, isopropanol and $n$-propanol) may cause skin dryness by dehydration and the non-alcohol based disinfectants (benzalkonium chloride and triclosan) may cause irritation on the skin and allergic reactions (Larson \& Morton, 1991; Basketter et al., 2004; USFDA, 2019). According to United States Food and Drug Administration (Weatherly \& Gosse, 2017), ethanol, benzalkonium chloride and triclosan are safe to use and allowed to be used as hand sanitizers with acceptable safety levels.

Herbal-based NAHS are widely recognised as natural and eco-friendly alternative to ABHS, benzalkonium chloride and triclosan based hand sanitizers. Many scientific investigators in the world have been extensively studied 
the potentiality of antibacterial, antifungal and antiviral activities of a number of medicinal and herbal species. However, information is scarce in the scientific literature data concerning the herbal-based NAHS with antimicrobial activities. During the past few decades, plant extracts/oils based bioactives were mixed with different supplementary ingredients and evaluated their efficacies as sanitizers against different species of most common microbial pathogens (Table 1). The following substances were reported as supplementary ingredients used for formulating herbal hand sanitizers; alcohol, aloe vera gel, carbomer, carbopol, coloring agents, deionized water, EDTA, emulsifier, excipients, fragrance, glycerine, methyl paraben, preservatives, propyl paraben, propylene glycol, sodium lauryl sulphate, solubilizer, triethanolamine and vinegar. Most of the herbal sanitizing agents were prepared with synergistic potential. However, the doses of extracts or oils have been represented in different forms of results (minimum inhibitory concentration, zone of inhibition, colony forming unit and log reduction) and units (\%, $\mathrm{mm}$ and $\mathrm{ml}$ ) discouraging comparison of the potentiality of sanitizing data. On the other hand, a number of herbal-based NAHS were formulated and now commercially available in the public market. Antimicrobial bioactives from Azadirachta indica, Cinnamomum zeylanicum Citrus limon, Cymbopogon citratus, Ocimum sanctum, Rosmarinus officinalis and Mentha piperita were most commonly used as technical ingredients in the commercialized sanitizer products. Currently, people are using those commercialized products with a strong belief of antiviral potential against COVID-19. On the other hand, at present it is a difficult task to investigate the antiviral potentialities of commercialized products and new formulations specifically against coronavirus. Likely, some of the sanitizer industrialists and scientific organizations have found an opportunity to suggest and transfer their formulations (ABHS and NAHS) and technologies with a presumption to combat the urgent need of current demands.

In ABHS formulations, ethanol and isopropyl alcohol are used as principal bioactives, glycerol and hydrogen peroxide $\left(\mathrm{H}_{2} \mathrm{O}_{2}\right)$ were incorporated as emollient and surfactant ingredients, respectively. Whereas, in herbal-based NAHS formulations, some other technical ingredients are additionally required to maintain the solubility, stability and bioactivity of herbal ingredient(s) (Figure 1). Accordingly, glycerin and isopropyl myristate were used as emollient, sodium lauryl ether sulfate, sodium lauryl sulphate and triethanolamine are used as surfactant, methyl paraben and aromatic essential oils are used as fragrances. Polysorbate20 is used as emulsifier, aloe vera extract, carbopol-940 and carbomer are used as gelling agents. EDTA, HPMC E-50, propylparaben, propylene glycol and vinegar are used as preservative agents. Acetone, ethanol and isopropyl alcohol are used as carrier/vehicles in herbal-based NAHS formulations. In addition to herbal bioactives, some of the technical ingredients such as EDTA, triethanolamine, sodium lauryl sulphate, propylparaben, propylene glycol, acetone, ethanol and isopropyl alcohol may also act as synergistic bioactives. Therefore, ethanol and/or isopropyl alcohol added herbal sanitizers might be called as herbal-based AHS.

Bacillus pumillus, Enterococcus faecalis, Enterococcus hirae, Escherichia coli, Micrococcus luteus, Propionibacterium acnes, Pseudomonas aeruginosa, Salmonella sp., Staphylococcus aureus, S. epidermidis and Klebsiella pneumonia are some of the bacterial species commonly found as pathogens on human skin. Recently, Kampf (2018) reviewed about the efficacy of ethanol as sanitizer on different types of viruses (adenovirus type 5 , poliovirus type 1 , murine norovirus, hepatitis $A$ virus, rotavirus and feline calicivirus) in-regard to hand disinfection. Based on the pathogenic epidermal microbiota, sanitizers have been investigated and their efficacies are reported by many microbiologists during the past decades. Furthermore, many investigators have extensively studied the potentiality of antiviral activities of herbal species against coxsackievirus, dengue virus, enterovirus, hepatitis $B$ virus, hepatitis C virus, herpes simplex virus (HSV), influenza virus, measles virus, respiratory syncytial virus and coronavirus (Lin et al., 2014). Most recently, WHO suggested to use ABHS to kill the COVID-19 (Vellingiri et al., 2020). In addition, herbal-based ABHS may be effective against corona virus due to the synergistic actions of alcohols with herbal bioactives. Further, addition of acids (citric acid, phosphoric acid or peracetic acid) to the formulations may enhance the antiviral activities (Kampf, 2018).

Gram-negative bacteria and enveloped virus have lipid bilayer that serves as a target of disinfectants. The SARSCoV2 contain single stranded RNA as genetic material that is surrounded by an envelope made of lipid bilayer ornamented with glycoprotein and spike proteins. According to Gold and Avva (2020), ethanol, isopropanol and propanol based ABHS are potential disinfectants against the most of the pathogenic viruses and bacteria. In the view of mechanistic actions, the alcohols (ethanol, isopropanol, $n$-propanol) have strong hydrogen bonding potential with lipids which leads to rapid protein denaturation in lipid bilayer membrane, and consequently leads to metabolism interference and cell lysis (McDonnell \& Russell, 1999). Thus, ABHS are acting as promising antibacterial and antivirals (including coronavirus) depending on the alcohol concentrations. On the other hand, plant essential oils are lipophilic in nature and the essential oil containing sanitizers may kill the bacteria by the disruption of bacterial membrane and cell lysis. Similarly, herbal-based NAHS may disrupt the lipid bilayer in the envelop of SARS-CoV2 and interfere with membrane proteins, which may lead to viral Iysis. Supportively, Astani et al. (2010) stated that essential oils and their monoterpene constituents may prevent the adsorption or entry of enveloped viruses into host cells by causing interference on the viral envelope structure. Recently, Thabti et al. (2021) stated that hydromethanolic extract of Morus alba inhibited the binding of enveloped human coronavirus (HCoV 229E) to the host cells by the binding of phenolic components with viral protein coat. Furthermore, according to Efimova and Ostroumova (2021), bioorganic saponins can be use as herbal surfactants with the capable of forming pores in model lipid membranes by lipid disordering and membrane curvature stress induction. When compared to enveloped viruses, herbals antiviral efficacies may be less in non-enveloped viruses because of lack of lipophilic interactions (Cermelli et al., 2008). Previously, Siddiqui et al. (1996) described about the dissolution of envelope in herpes simplex virus type 1 and newcastle disease virus due to the antiviral action of essential oils. Most recently, Nadjib (2020) reviewed about the mechanistic action of essential oils and terpenes as antivirals on the coronaviruses. In view of the above facts, at present and in future the antibacterial hand sanitizers including herbal products may be used as antiviral disinfectant for the management of COVID-19.

\section{Conflict of interest}

The authors declare no conflicts of interest. 
Madhurya et al. (2021), Tropical Biomedicine 38(4): 605-612

Table 1. Herbal extracts as sanitizing agents and antimicrobial efficacies against most common microbial pathogens

\begin{tabular}{|c|c|c|c|c|}
\hline \multicolumn{2}{|c|}{ Herbal Hand Sanitizer } & \multirow[b]{2}{*}{ Tested Organisms } & \multirow[b]{2}{*}{ Activity } & \multirow[b]{2}{*}{ Reference } \\
\hline $\begin{array}{l}\text { Bioactives } \\
\text { (Mixture / Individual) }\end{array}$ & Ingredients & & & \\
\hline $\begin{array}{l}\text { Citrus aurantifolia } \\
\text { C. citratus }\end{array}$ & $\begin{array}{l}\text { Hydroxypropyl } \\
\text { methylcellulose } \\
\text { Triethanolamine } \\
\text { Propylene glycol } \\
\text { Aquadest }\end{array}$ & Staphylococcus aureus & $\mathrm{ZI}: 7.00 \mathrm{~mm}$ & Djima et al. (2021) \\
\hline Syzygium aromaticum & & $\begin{array}{l}\text { Acinetobacter baumannii } \\
\text { Escherichia coli } \\
\text { Klebsiella pneumoniae } \\
\text { Pseudomonas aeruginosa } \\
\text { Staphylococcus aureus } \\
\text { S. epidermidis } \\
\text { S. hominis } \\
\text { S. haemolyticus } \\
\text { Micrococcus luteus } \\
\text { Candida albicans }\end{array}$ & $\begin{array}{l}\text { ZI : } 9.00 \mathrm{~mm} \\
\text { ZI : } 8.00 \mathrm{~mm} \\
\text { ZI : } 9.00 \mathrm{~mm} \\
\text { ZI : } 9.00 \mathrm{~mm} \\
\mathrm{ZI}: 13.00 \mathrm{~mm} \\
\mathrm{ZI}: 9.00 \mathrm{~mm} \\
\mathrm{ZI}: 9.00 \mathrm{~mm} \\
\mathrm{ZI}: 13.00 \mathrm{~mm} \\
\mathrm{ZI}: 9.00 \mathrm{~mm} \\
\mathrm{ZI}: 7.00 \mathrm{~mm}\end{array}$ & \\
\hline Lavendula sp. & $\begin{array}{l}\text { Aloe vera gel } \\
\text { Glycerin } \\
\text { Vitamin } \mathrm{E}\end{array}$ & $\begin{array}{l}\text { Acinetobacter baumannii } \\
\text { Escherichia coli } \\
\text { Klebsiella pneumoniae } \\
\text { Pseudomonas aeruginosa } \\
\text { Staphylococcus aureus } \\
\text { S. epidermidis } \\
\text { S. hominis } \\
\text { S. haemolyticus } \\
\text { Micrococcus luteus } \\
\text { Candida albicans }\end{array}$ & $\begin{array}{l}\text { ZI : } 8.00 \mathrm{~mm} \\
\text { ZI : } 8.00 \mathrm{~mm} \\
\text { ZI : } 8.00 \mathrm{~mm} \\
\text { ZI : } 8.00 \mathrm{~mm} \\
\mathrm{ZI}: 9.00 \mathrm{~mm} \\
\mathrm{ZI}: 8.00 \mathrm{~mm} \\
\mathrm{ZI}: 7.00 \mathrm{~mm} \\
\mathrm{ZI}: 7.00 \mathrm{~mm} \\
\mathrm{ZI}: 9.00 \mathrm{~mm} \\
\mathrm{ZI}: 0.00 \mathrm{~mm}\end{array}$ & Booq et al. (2021) \\
\hline Melaleuca alternifolia & & $\begin{array}{l}\text { Acinetobacter baumannii } \\
\text { Escherichia coli } \\
\text { Klebsiella pneumoniae } \\
\text { Pseudomonas aeruginosa } \\
\text { Staphylococcus aureus } \\
\text { S. epidermidis } \\
\text { S. hominis } \\
\text { S. haemolyticus } \\
\text { Micrococcus luteus } \\
\text { Candida albicans }\end{array}$ & $\begin{array}{l}\text { ZI : } 18.00 \mathrm{~mm} \\
\text { ZI : } 9.00 \mathrm{~mm} \\
\text { ZI : } 12.00 \mathrm{~mm} \\
\text { ZI : } 12.00 \mathrm{~mm} \\
\text { ZI : } 17.00 \mathrm{~mm} \\
\text { ZI : } 20.00 \mathrm{~mm} \\
\text { ZI : } 13.00 \mathrm{~mm} \\
\text { ZI : } 12.00 \mathrm{~mm} \\
\text { ZI : } 17.00 \mathrm{~mm} \\
\text { ZI : } 0.00 \mathrm{~mm}\end{array}$ & \\
\hline $\begin{array}{l}\text { Piper betle } \\
\text { Citrus aurantifolia }\end{array}$ & Distilled water & $\begin{array}{l}\text { Staphylococcus aureus } \\
\text { Escherichia coli }\end{array}$ & $\begin{array}{l}\mathrm{ZI}: 11.00 \mathrm{~mm} \\
\mathrm{ZI}: 11.10 \mathrm{~mm}\end{array}$ & Gloria et al. (2021) \\
\hline $\begin{array}{l}\text { Curcuma longa } \\
\text { Azadirachta indica }\end{array}$ & $\begin{array}{l}\text { Carbapol-940 } \\
\text { Glycerine } \\
\text { Hydroxypropyl } \\
\text { methylcellulose-E15 } \\
\text { Perfuming agent } \\
\text { Sodium hydroxide }\end{array}$ & $\begin{array}{l}\text { Bacillus subtilis } \\
\text { Escherichia coli }\end{array}$ & $\begin{array}{l}\mathrm{ZI}: 15.00 \mathrm{~mm} \\
\mathrm{ZI}: 11.10 \mathrm{~mm}\end{array}$ & Maddi et al. (2021) \\
\hline $\begin{array}{l}\text { Tea extract } \\
\text { Cymbopogon sp. }\end{array}$ & $\begin{array}{l}\text { Alcohol } \\
\text { Glycerol } \\
\text { Hydrogen peroxide }\end{array}$ & $\begin{array}{l}\text { Escherichia coli } \\
\text { Klebsiella pneumoniae } \\
\text { Micrococcus luteus } \\
\text { Salmonella typhi }\end{array}$ & $\begin{array}{l}\mathrm{ZI}: 4.00 \mathrm{~mm} \\
\mathrm{ZI}: 11.00 \mathrm{~mm} \\
\mathrm{ZI}: 9.66 \mathrm{~mm} \\
\mathrm{ZI}: 9.00 \mathrm{~mm}\end{array}$ & Rana et al. (2021) \\
\hline
\end{tabular}




\begin{tabular}{|c|c|c|c|c|}
\hline & & Staphylococcus aureus & $\begin{array}{l}\mathrm{ZI}: 13.33 \mathrm{~mm} \\
\mathrm{ZI}: 21.66 \mathrm{~mm}\end{array}$ & \\
\hline $\begin{array}{l}\text { Azadirachta indica } \\
\text { Eucalyptus globulus }\end{array}$ & $\begin{array}{l}\text { Alcohol } \\
\text { Methyl paraben } \\
\text { Perfume } \\
\text { Polysorbate } 20 \\
\text { Sorbitol }\end{array}$ & $\begin{array}{l}\text { Bacillus subtilis } \\
\text { Escherichia coli } \\
\text { Pseudomonas aeurogenosa } \\
\text { Staphylococcus aureus }\end{array}$ & $\begin{array}{l}\mathrm{ZI}: 30.00 \mathrm{~mm} \\
\mathrm{ZI}: 32.00 \mathrm{~mm} \\
\mathrm{ZI}: 34.00 \mathrm{~mm} \\
\mathrm{ZI}: 34.00 \mathrm{~mm}\end{array}$ & $\begin{array}{l}\text { Surwase et al. } \\
\text { (2021) }\end{array}$ \\
\hline $\begin{array}{l}\text { Citus sinensis } \\
\text { Eucalyptus globulus }\end{array}$ & $\begin{array}{l}\text { Alcohol } \\
\text { Glycerol } \\
\text { Hydrogen peroxide }\end{array}$ & $\begin{array}{l}\text { Escherichia coli } \\
\text { Staphylococcus aureus }\end{array}$ & $\begin{array}{l}\mathrm{ZI}: 22.00 \mathrm{~mm} \\
\mathrm{ZI}: 26.00 \mathrm{~mm}\end{array}$ & $\begin{array}{l}\text { Suryawanshi et al. } \\
(2020)\end{array}$ \\
\hline $\begin{array}{l}\text { Aloe barbadensis } \\
\text { Azadirachta indica } \\
\text { Ocimum sanctum }\end{array}$ & $\begin{array}{l}\text { Coloring agents } \\
\text { Excipients } \\
\text { Fragrances } \\
\text { Isopropanol } \\
\text { Preservatives }\end{array}$ & $\begin{array}{l}\text { Staphylococcus aureus } \\
\text { S. epidermidis }\end{array}$ & $\begin{array}{l}\text { MIC : } 0.03 \% \\
\text { MIC : } 0.02 \%\end{array}$ & $\begin{array}{l}\text { Balkrishna et al. } \\
(2020)\end{array}$ \\
\hline $\begin{array}{l}\text { Azadirachta indica } \\
\text { Citrus aurantiifolia } \\
\text { Coleus zeylanicus } \\
\text { Coriandrum sativum } \\
\text { Vetiveria zizanioides }\end{array}$ & - & $\begin{array}{l}\text { Escherichia coli } \\
\text { Psuedomonas aeruginosa } \\
\text { Staphylococcus aureus }\end{array}$ & $\begin{array}{l}\text { MIC : } 50.00 \% \\
\text { MIC : } 50.00 \% \\
\text { MIC : } 50.00 \%\end{array}$ & Goodarzi, (2020) \\
\hline Citrus aurantifolia & $\begin{array}{l}\text { Alcohol } \\
\text { Carbopol } \\
\text { Deionized water } \\
\text { Glycerine } \\
\text { Triethanolamine }\end{array}$ & $\begin{array}{l}\text { Bacillus subtilis } \\
\text { Candida albicans } \\
\text { Escherichia coli } \\
\text { Psuedomonas aeruginosa } \\
\text { Salmonella typhi } \\
\text { Staphylococcus aureus }\end{array}$ & $\begin{array}{l}\text { ZI : } 20.50 \mathrm{~mm} \\
\mathrm{ZI}: 19.00 \mathrm{~mm} \\
\mathrm{ZI}: 15.50 \mathrm{~mm} \\
\mathrm{ZI}: 24.50 \mathrm{~mm} \\
\mathrm{ZI}: 19.00 \mathrm{~mm} \\
\mathrm{ZI}: 31.50 \mathrm{~mm}\end{array}$ & $\begin{array}{l}\text { Odimegwu et al. } \\
(2020)\end{array}$ \\
\hline $\begin{array}{l}\text { Aloe vera } \\
\text { Azadirachta indica } \\
\text { Bixa sp. } \\
\text { Citrus limon } \\
\text { Coriandrum sativum } \\
\text { Rosa sp. }\end{array}$ & $\begin{array}{l}\text { Alcohol } \\
\text { Glycerine } \\
\text { Sodium lauryl sulphate }\end{array}$ & $\begin{array}{l}\text { Escherichia coli } \\
\text { Psuedomonas aeruginosa } \\
\text { Staphylococcus aureus }\end{array}$ & $\begin{array}{l}\mathrm{ZI}: 28.00 \mathrm{~mm} \\
\mathrm{ZI}: 15.00 \mathrm{~mm} \\
\mathrm{ZI}: 23.00 \mathrm{~mm}\end{array}$ & Chandrudu, (2019) \\
\hline $\begin{array}{l}\text { Azadirachta indica } \\
\text { Catharanthus roseus } \\
\text { Eucalyptus sp. }\end{array}$ & Glycerine & Finger microflora & CFU : 30 & $\begin{array}{l}\text { Singla \& Saini } \\
(2019)\end{array}$ \\
\hline $\begin{array}{l}\text { Coccus nucifera } \\
\text { Morus alba }\end{array}$ & $\begin{array}{l}\text { Emulsifier } \\
\text { Glycerine } \\
\text { Solubilizer }\end{array}$ & $\begin{array}{l}\text { Staphylococcus sp. } \\
\text { Streptococcus sp. }\end{array}$ & $\begin{array}{l}\mathrm{ZI}: 14.50 \mathrm{~mm} \\
\mathrm{ZI}: 14.00 \mathrm{~mm}\end{array}$ & Jusoh et al. (2019) \\
\hline Melaleuca alternifolia & & Finger microflora & CFU : 13 & Alobaid et al. (2019) \\
\hline Ficus lyrate & $\begin{array}{l}\text { Alcohol } \\
\text { Carbopol } 940 \\
\text { Distilled water } \\
\text { Glycerine } \\
\text { Methylparaben } \\
\text { Triethanolamine }\end{array}$ & Escherichia coli & $\mathrm{CFU} / \mathrm{ml}: 3$ & Wira et al. (2019) \\
\hline Azadirachta indica & \multirow{3}{*}{$\begin{array}{l}\text { Glycerol } \\
\text { Isopropanol } \\
\text { Rose water }\end{array}$} & $\begin{array}{l}\text { Candida albicans } \\
\text { Hand swab isolates } \\
\text { MRSA } \\
\text { Psuedomonas aeruginosa } \\
\text { Staphylococcus aureus }\end{array}$ & $\begin{array}{l}\text { ZI : } 15.00 \mathrm{~mm} \\
\text { Zl : } 28.00 \mathrm{~mm} \\
\text { ZI : } 23.00 \mathrm{~mm} \\
\text { ZI : } 15.00 \mathrm{~mm} \\
\text { ZI : } 25.00 \mathrm{~mm}\end{array}$ & \multirow{3}{*}{$\begin{array}{l}\text { Patankar \& } \\
\text { Chandak (2018) }\end{array}$} \\
\hline Citrus limon & & $\begin{array}{l}\text { Candida albicans } \\
\text { Hand swab isolates } \\
\text { MRSA } \\
\text { Staphylococcus aureus }\end{array}$ & $\begin{array}{l}\mathrm{ZI}: 12.00 \mathrm{~mm} \\
\mathrm{ZI}: 32.00 \mathrm{~mm} \\
\mathrm{ZI}: 32.00 \mathrm{~mm} \\
\mathrm{ZI}: 24.00 \mathrm{~mm}\end{array}$ & \\
\hline $\begin{array}{l}\text { Azadirachta indica } \\
\text { Citrus limon }\end{array}$ & & $\begin{array}{l}\text { Candida albicans } \\
\text { Hand swab isolates } \\
\text { MRSA } \\
\text { Psuedomonas aeruginosa } \\
\text { Staphylococcus aureus }\end{array}$ & $\begin{array}{l}\mathrm{ZI}: 10.00 \mathrm{~mm} \\
\mathrm{ZI}: 34.00 \mathrm{~mm} \\
\mathrm{ZI}: 28.00 \mathrm{~mm} \\
\mathrm{ZI}: 14.00 \mathrm{~mm} \\
\mathrm{ZI}: 24.00 \mathrm{~mm}\end{array}$ & \\
\hline
\end{tabular}




\begin{tabular}{|c|c|c|c|c|}
\hline $\begin{array}{l}\text { Azadirachta indica } \\
\text { Citrus limon } \\
\text { Ocimum sanctum }\end{array}$ & $\begin{array}{l}\text { Carbapol-940 } \\
\text { Deionized water } \\
\text { EDTA } \\
\text { Glycerine } \\
\text { Perfume }\end{array}$ & $\begin{array}{l}\text { Candida albicans } \\
\text { Escherichia coli } \\
\text { Salmonella sp. } \\
\text { Staphylococcus aureus }\end{array}$ & - & $\begin{array}{l}\text { Acharya et al. } \\
\text { (2018) }\end{array}$ \\
\hline Syzygium polyanthum & $\begin{array}{l}\text { Aqua destillata } \\
\text { Carbomer } \\
\text { Clove fragrance } \\
\text { Isopropyl myristate } \\
\text { Propylene glycol } \\
\text { Triethanolamine }\end{array}$ & Staphylococcus aureus & MIC : $3.12 \%$ & Surini et al. (2018) \\
\hline Azadirachta indica & \multirow{3}{*}{$\begin{array}{l}\text { Deionized water } \\
\text { Glycerine } \\
\text { Carbopol } 940 \\
\text { Perfume } \\
\text { Polysorbate } 20 \\
\text { Triethanolamine } \\
\text { Alcohol }\end{array}$} & $\begin{array}{l}\text { Escherichia coli } \\
\text { Staphylococcus aureus }\end{array}$ & $\begin{array}{l}\mathrm{ZI}: 15.00 \mathrm{~mm} \\
\mathrm{ZI}: 17.60 \mathrm{~mm}\end{array}$ & \multirow{3}{*}{ Shah et al. (2018) } \\
\hline Ocimum sanctum & & $\begin{array}{l}\text { Escherichia coli } \\
\text { Staphylococcus aureus }\end{array}$ & $\begin{array}{l}\mathrm{Zl}: 17.60 \mathrm{~mm} \\
\mathrm{ZI}: 15.30 \mathrm{~mm}\end{array}$ & \\
\hline Zingiber officinale & & $\begin{array}{l}\text { Escherichia coli } \\
\text { Staphylococcus aureus }\end{array}$ & $\begin{array}{l}\mathrm{ZI}: 14.30 \mathrm{~mm} \\
\mathrm{ZI}: 17.00 \mathrm{~mm}\end{array}$ & \\
\hline Annona Muricata & $\begin{array}{l}\text { Aqua destillata } \\
\text { Carbopol } \\
\text { Glycerine } \\
\text { Methyl Paraben } \\
\text { Triethanolamine }\end{array}$ & Propionibacterium acnes & $\mathrm{Zl}: 3.53 \mathrm{~mm}$ & $\begin{array}{l}\text { Ningsih et al. } \\
\text { (2017) }\end{array}$ \\
\hline $\begin{array}{l}\text { Cassia fistula } \\
\text { Ficus religiosa } \\
\text { Milletia pinnata }\end{array}$ & $\begin{array}{l}\text { Alcohol } \\
\text { Carbapol } \\
\text { Cinnamon oil } \\
\text { Citronella oil } \\
\text { Glycerine } \\
\text { Methyl paraben } \\
\text { Perfume } \\
\text { Polysorbate-20 } \\
\text { Triethanolamine } \\
\text { Water }\end{array}$ & $\begin{array}{l}\text { Escherichia coli } \\
\text { Pseudomonas aeruginosa } \\
\text { Staphylococcus aureus }\end{array}$ & $\begin{array}{l}\mathrm{ZI}: 26.00 \mathrm{~mm} \\
\mathrm{ZI}: 24.00 \mathrm{~mm} \\
\mathrm{ZI}: 22.00 \mathrm{~mm}\end{array}$ & $\begin{array}{l}\text { Afsar \& Khanam } \\
\text { (2016) }\end{array}$ \\
\hline \multirow{2}{*}{$\begin{array}{l}\text { Azadirachta indica } \\
\text { Cinnamomum camphor } \\
\text { Citrus limon } \\
\text { Cuminum cyminum } \\
\text { Vetiveria zizaniodes }\end{array}$} & & $\begin{array}{l}\text { Aspergillus niger } \\
\text { Candida albicans }\end{array}$ & $\begin{array}{l}\text { LR }: 0.11 \\
\text { LR }: 0.63\end{array}$ & $\begin{array}{l}\text { Harsha et al. } \\
\text { (2016a) }\end{array}$ \\
\hline & $\begin{array}{l}\text { Base-IPA } \\
\text { Perfume }\end{array}$ & $\begin{array}{l}\text { Enterococcus hirae } \\
\text { Escherichia coli } \\
\text { Pseudomonas aeruginosa } \\
\text { Staphylococcus aureus }\end{array}$ & $\begin{array}{l}\text { LR }: 0.09 \\
\text { LR }: 0.10 \\
\text { LR }: 0.13 \\
\text { LR }: 0.03\end{array}$ & $\begin{array}{l}\text { Harsha et al. } \\
(2016 b)\end{array}$ \\
\hline $\begin{array}{l}\text { Azadirachta indica } \\
\text { Cariandrum sativum } \\
\text { Citrus aurantiifolia } \\
\text { Pavonia odarata } \\
\text { Vetiveria zizaniodes }\end{array}$ & - & $\begin{array}{l}\text { Enterococcus faecalis } \\
\text { Escherichia coli } \\
\text { Pseudomonas aeruginosa } \\
\text { Staphylococcus aureus } \\
\text { S. epidermidis }\end{array}$ & $\begin{array}{l}\text { Zl : } 7.00 \mathrm{~mm} \\
\text { ZI : } 7.50 \mathrm{~mm} \\
\mathrm{ZI}: 7.50 \mathrm{~mm} \\
\mathrm{ZI}: 3.50 \mathrm{~mm} \\
\mathrm{ZI}: 7.00 \mathrm{~mm}\end{array}$ & Jain et al. (2016) \\
\hline $\begin{array}{l}\text { Aegle marmelos } \\
\text { Azadirachta indica } \\
\text { Cinnamomum verum } \\
\text { Ocimum tenuiflorum } \\
\text { Phyllanthus niruri } \\
\text { Syzygium aromaticum }\end{array}$ & $\begin{array}{l}\text { Aloe vera gel } \\
\text { Vinegar }\end{array}$ & $\begin{array}{l}\text { Escherichia coli } \\
\text { Pseudomonas aeruginosa }\end{array}$ & - & Rajurkar (2016) \\
\hline $\begin{array}{l}\text { Citrus limon } \\
\text { Cymbopogon citratus } \\
\text { Eucalyptus }\end{array}$ & - & Palm microflora & - & $\begin{array}{l}\text { Ramesh et al. } \\
\text { (2016) }\end{array}$ \\
\hline $\begin{array}{l}\text { Ficus bengalensis } \\
\text { F. glomerata } \\
\text { F. lecor } \\
\text { F. religiosa } \\
\text { Thespesia populnea }\end{array}$ & $\begin{array}{l}\text { Carbopol } \\
\text { Methyl paraben } \\
\text { Perfume } \\
\text { Propyl paraben } \\
\text { Sodium lauryl ether } \\
\text { sulfate } \\
\text { Triethanolamine }\end{array}$ & $\begin{array}{l}\text { Bacillus pumillus } \\
\text { Staphylococcus aureus }\end{array}$ & $\begin{array}{l}\mathrm{Zl}: 19.20 \mathrm{~mm} \\
\mathrm{ZI}: 17.00 \mathrm{~mm}\end{array}$ & Vyas et al. (2011) \\
\hline
\end{tabular}

MIC - Minimum Inhibitory Concentration, ZI - Zone of Inhibition, CFU - Colony Forming Unit, LR - Log reduction, MRSA - MethicillinResistant Staphylococcus aureus. 


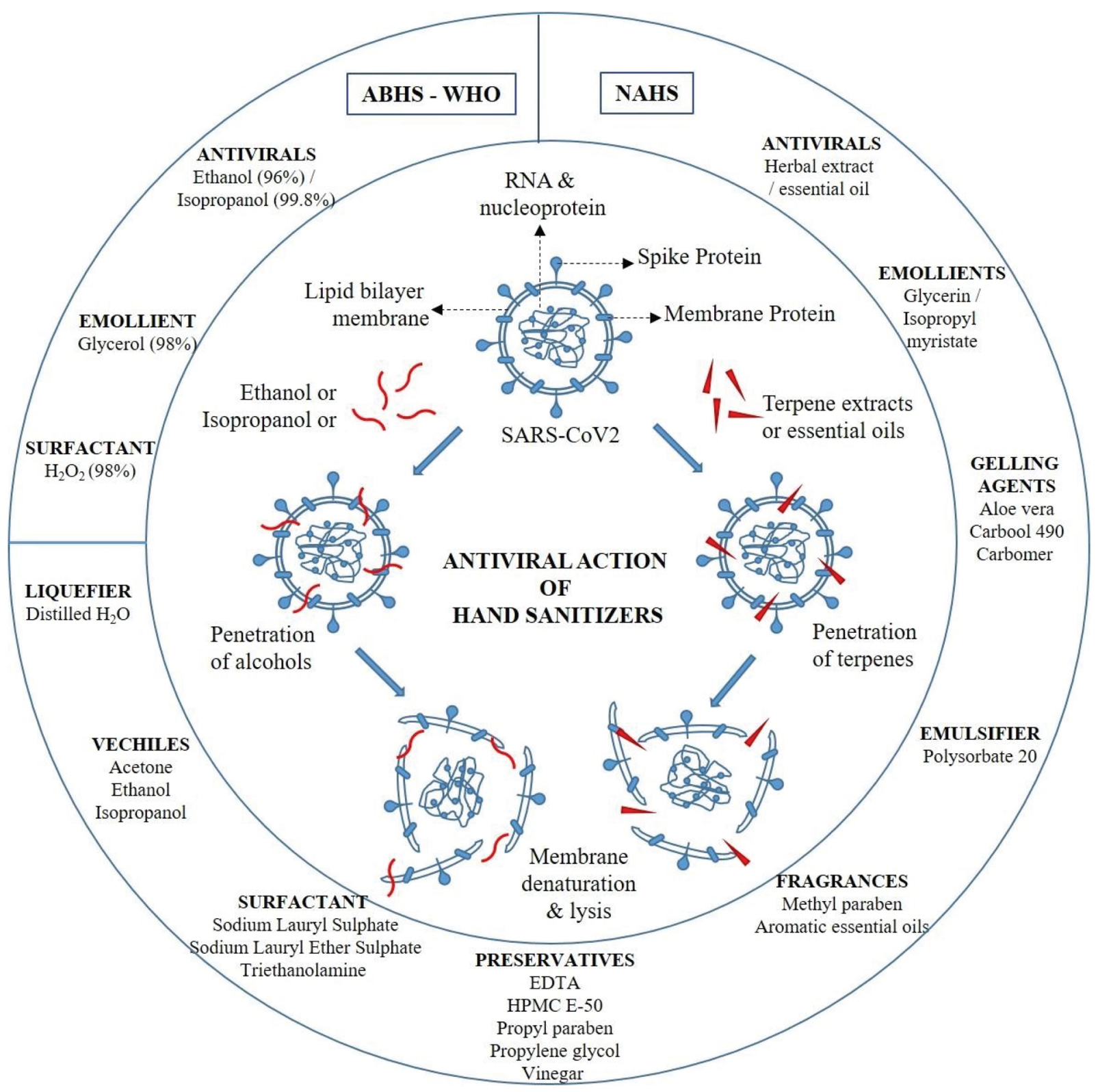

Figure 1. Ingredients of hand sanitizers and antiviral actions. ABHS-WHO: Alcohol based hand sanitizer-World Health Organization, NAHS - Non-alcohol based hand sanitizer, RNA - Ribonucleic acid, EDTA - Ethylenediaminetetraacetic acid, HPMC E-50 - Hydroxy propyl methyl cellulose E-50.

\section{REFERENCES}

Acharya, S.B., Ghosh, S., Yadav, G., Sharma, K., Ghosh, S. \& Joshi, S. (2018). Formulation, evaluation and antibacterial efficiency of water-based herbal hand sanitizer gel. BioRxiv 373928 [Preprint]. https://doi.org/10.1101/373928

Afsar, Z. \& Khanam, S. (2016). Formulation and evaluation of poly herbal soap and hand sanitizer. International Research Journal of Pharmacy 7: 54-57. https://doi.org/10.7897/22308407.07896

Alobaid, A.S., Fadul, A.N., Asiri, M.N., Asiri, E.M., Alqahtani, W.M. \& Alshareef, N.Y. (2019). Antiseptic ability of tea tree oil in hand hygiene among dental students attending King Khalid University. EC Dental Science 18: 129-135.
Astani, A., Reichling, J. \& Schnitzler, P. (2010). Comparative study on the antiviral activity of selected monoterpenes derived from essential oils. Phytotherapy Research 24: 673679. https://doi.org/10.1002/ptr.2955

Balkrishna, A., Singh, K., Singh, H., Haldar, S. \& Varshney, A. (2020). GermiX: a skin friendly hand sanitizer with prolonged effectivity against pathogenic bacteria. $A M B$ Express 10: 210. https://doi.org/10.1186/s13568-02001151-y

Basketter, D.A., Marriott, M., Gilmour, N.J. \& White, I.R. (2004). Strong irritants masquerading as skin allergens: the case of benzalkonium chloride. Contact Dermatitis 50: 213-217. https://doi.org/10.1111/j.0105-1873.2004.00331.x 
Booq, R.Y., Alshehri, A.A., Almughem, F.A., Zaidan, N.M., Aburayan, W.S., Bakr, A.A., Kabli, S.H., Alshaya, H.A., Alsuabeyl, M.S., Alyamani, E.J. et al. (2021). Formulation and evaluation of alcohol-free hand sanitizer gels to prevent the spread of infections during pandemics. International Journal of Environmental Research and Public Health 18: 6252. https://doi.org/10.3390/ijerph18126252

Cermelli, C., Fabio, A., Fabio, G. \& Quaglio, P. (2008). Effect of eucalyptus essential oil on respiratory bacteria and viruses. Current Microbiology 56: 89-92. https://doi.org/ 10.1007/s00284-007-9045-0

Chandrudu, J. (2019). Formulation and standardization of polyherbal hand sanitizer. International Journal of Pharmaceutical Research and Life Sciences 7: 10-13

Djima, E.G.E., Prasetyaningsih, A. \& Madyaningrana, K. (2021). Antibacterial activity of lime peel and lemongrass extract as active ingredients for spray hand sanitizer. SCISCITATIO Journal of Biological Science 2: 22-28.

Efimova, S.S. \& Ostroumova, O.S. (2021). Is the membrane lipid matrix a key target for action of pharmacologically active plant saponins? International Journal of Molecular Sciences 22: 3167. https://doi.org/10.3390/ijms22063167

Gloria, R.Y., Yuliyani, R. \& Asror, M.M.S. (2021). Effectiveness of green betel leaf and lime extract against Staphylococcus aureus and Escherichia coli. Biodiversitas 22: 3452-3457. https://doi.org/10.13057/biodiv/d220843

Gold, N.A., Mirza T.M. \& Avva, U. (2020). Alcohol sanitizer. In: StatPearls [Internet]. Treasure Island (FL): StatPearls Publishing.

Goodarzi, A. (2020). COVID -19 and estimate of antimicrobial efficacy of hand sanitizers. Global Journal of Pathology and Microbiology 8: 6-11.

Harsha, M.R., Mishra, B., Chaithra, C.S. \& Ramana, V. (2016a). Evaluation of fungicidal activity of herbal hand sanitizer. Journal of Research in Traditional Medicine 2: 70-74

Harsha, M.R., Mishra, B., Chaithra, C.S. \& Ramana, V. (2016b). Evaluation of bactericidal activity of herbal hand sanitizer. International Journal of Ayurveda and Pharma Research 4: 24-28

Jain, V.M., Karibasappa, G.N., Dodamani, A.S., Prashanth, V.K. \& Mali, G.V. (2016). Comparative assessment of antimicrobial efficacy of different hand sanitizers: an in vitro study. Dental Research Journal (Isfahan) 13: 424-431. https:// doi.org/10.4103/1735-3327.192283

Jusoh, N., Zulkifli, F.Z.A., Zulkipli, H., Mahbob, E.N.M. \& Rosman, P.S. (2019). Synergism of virgin coconut oil and mulberry leaves extract as agent in free alcohol hand sanitizer. 2nd Kelantan International Learning and Innovation Exhibition UiTM Kelantan, Malaysia

Kampf, G. (2018). Efficacy of ethanol against viruses in hand disinfection. Journal of Hospital Infection 98: 331-338. https://doi.org/10.1016/j.jhin.2017.08.025

Larson, E.L. \& Morton, H.E. (1991). Alcohols. In: Disinfection, Sterilization, and Preservation, Block, S.S. (editor) 4th edition. Philadelphia: Lea \& Febiger, pp. 191-203.

Lin, L.T., Hsu, W.C. \& Lin, C.C. (2014). Antiviral natural products and herbal medicines. Journal of Traditional and Complement Medicine 4: 24-35. https://dx.doi.org/10.4103\%2F22254110.124335

Maddi, R., Perumalla, S., Sesham, L.K., Shaik, S.S., Tanniru, J., Uppu, H.B., Kalasani, S.L., Begum, A. \& Nadendla, R.R. (2021). Formulation and evaluation of curcumin gel sanitizer. Journal of Drug Delivery and Therapeutics 11: 64-70. http://doi.org/10.22270/jddt.v11i4-S.4985

McDonnell, G. \& Russell, A.D. (1999). Antiseptics and disinfectants: activity, action, and resistance. Clinical Microbiology Reviews 12: 147-179. https://doi.org/10.1128/ CMR.12.1.147
Nadjib, B.M. (2020). Effective antiviral activity of essential oils and their characteristic terpenes against coronaviruses: an update. Journal of Pharmacology and Clinical Toxicology 8: 1138.

Ningsih, D.R., Zusfahair, Kartika, D. \& Fatoni, A. (2017). Formulation of hand sanitizer with antibacterials substance from $\mathrm{n}$-hexane extract of soursop leaves (Annona muricata Linn). Malaysian Journal of Fundamental and Applied Science 13: 1-5. https://doi.org/10.11113/ mjfas.v13n1.527

Odimegwu, J.I., Adegbaju, B.E. \& Ilomuanya, M.O. (2020). Citrutox ${ }^{\circledast}$ Hand Sanitizer - an innovative essential oil and alcohol-based sanitizer for preventing COVID-19 infections. Journal of Basic Social Pharmacy Research 1: S28S41.

Patankar, R.S. \& Chandak, N. (2018). Formulation of herbal sanitizers and determining their antimicrobial activities against skin pathogens. International Journal of Innovative Science and Research Technology 3: 169-177.

Rajurkar, V.H. (2016). Synthesis and characterization of non alcoholic hand washer by using natural herbs. International Journal of Science and Research 5: 47-48.

Ramesh, G., Seth, R.K., Sujatha, R. \& Chaubey, S. (2016). Evaluation of antibacterial efficacy of lemon grass oil eucalyptus oil and lemon juice, as a hand sanitizer. International Ayurvedic Medical Journal 4: 1193-1203.

Rana, A., Mukhia, S., Acharya, R. \& Kumar, S. (2021). Hand sanitizer with natural ingredients exhibits enhanced antimicrobial efficacy. Research Square [Preprint]. https:// doi.org/10.21203/rs.3.rs-357976/v1

Shah, S.K., Banode, V.R., Gholse, Y.N. \& Chaple, D.R. (2018). Extraction, formulation and evaluation of polyherbal hand sanitizer. Research Pharmaceutica 2: 01-03.

Siddiqui, Y.M., Ettayebi, M., Haddad, M.D.L., Ahdal, M.N. \& Haddad, A.E. (1996). Effect of essential oils on the enveloped viruses: antiviral activity of oregano and clove oils on herpes simplex virus type 1 and Newcastle disease virus. Medical Science Research 24: 185-186.

Singla, D. \& Saini, K. (2019). Formulation of an herbal substitute for chemical sanitizer and its evaluation for antimicrobial efficiency. International Journal of ChemTech Research 12: 114-120. https://doi.org/10.20902/IJCTR.2019. 120318

Surini, S., Amirtha, N.I. \& Lestari, D.C. (2018). Formulation and effectiveness of a hand sanitizer gel produced using salam bark extract. International Journal of Applied Pharmaceutics 10: 216-220. http://doi.org/10.22159/ ijap.2018.v10s1.48

Surwase, V.B., Savale, M.M., Jadhav, R.J., Kadam, A.B. \& Shinde, P.P. (2021). Polyherbal natural hand sanitizer formulation and evaluation. Journal of University of Shanghai for Science and Technology 23: 932-939.

Suryawanshi, N.R., Surani, H.C. \& Yadav, H.R. (2020). Formulation, evaluation and anti-microbial efficiency of alcohol based herbal hand sanitizer. International Journal of Engineering Science and Computing 10: 25113-25115.

Thabti, I., Albert, Q., Philippot, S., Dupire, F., Westerhuis, B., Fontanay, S., Risler, A., Kassab, T., Elfalleh, W., Aferchichi, A. et al. (2020). Advances on antiviral activity of Morus spp. plant extracts: human coronavirus and virus-related respiratory tract infections in the spotlight. Molecules 25: 1876. https://doi.org/10.3390/molecules 25081876

USFDA. (2019). FDA issues final rule on safety and effectiveness of consumer hand sanitizers. FDA News Release. https: / / www.fda.gov/news-events/pressannouncements/fda-issues-final-rule-safety-andeffectiveness-consumer-hand-sanitizers. Accessed 16 December 2021. 
Vellingiri, B., Jayaramayya, K., Iyer, M., Narayanasamy, A., Govindasamy, V., Giridharan, B., Ganesan, S., Venugopal, A., Venkatsan, D., Ganesan, H. et al. (2020). COVID-19: A promising cure for the global panic. Science of the Total Environment 725: 138277. https://doi.org/10.1016/j. scitotenv.2020.138277

Vyas, P., Galib. Patgiri, B.J. \& Prajapati, P.K. (2011). Antimicrobial activity of ayurvedic hand sanitizers. International Journal of Pharmaceutical and Biological Archive 2: 762-766.

Weatherly, L.M. \& Gosse, J.A. (2017). Triclosan exposure, transformation, and human health effects. Journal of Toxicology and Environmental Health B 20: 447-469. https:// doi.org/10.1080\%2F10937404.2017.1399306

WHO. (2010). Guide to local production: WHO-recommended handrub formulations. https://www.who.int/gpsc/5may/ Guide_to_Local_Production.pdf.
WHO. (2021). Tracking SARS-CoV-2 variants. https://www.who. int/en/activities/tracking-SARS-CoV-2-variants. Accessed 16 December 2021.

Wilder-Smith, A., Chiew, C.J. \& Lee, V.J. (2020). Can we contain the COVID-19 outbreak with the same measures as for SARS? The Lancet Infectious Diseases 20: 102-107. https:// doi.org/10.1016/s1473-3099(20)30129-8

Wira, D., Putri, S., Mardawati, E., Kamila, H. \& Balia, R. (2019). Characterization and antibacterial activity test of hand sanitizer gel ethanol extract Ficus lyrata Warb against Escherichia coli Bacteria. Advances in Health Sciences Research 19: 59-62. https://doi.org/10.2991/isessah-19.2019.17 